\title{
Numerical Evaluation of Ultrasonic Pulse-echo Subwavelength Defect Detection
}

\author{
Xiangtao Yin ${ }^{* 1}$, Scott A. Morris ${ }^{\dagger}$ and William D. O'Brien, Jr. \\ *Bioacoustics Research Laboratory, Department of Electrical and Computer Engineering, University of Illinois \\ at Urbana-Champaign, 405 N. Mathews, Urbana, IL 61801 and $\dagger$ Department of Food Science and Human \\ Nutrition, University of Illinois at Urbana-Champaign, 1304 W. Pennsylvania Avenue, Urbana, IL 61801
}

\begin{abstract}
The ultrasonic pulse-echo Backscattered Amplitude Integral (BAI)-mode imaging technique [UFFC Trans, 45:30, 1998] has been developed to detect small channel defects in flexible food package seals. This technique detects 38- $\mu m$-diameter channels reliably and 6- $\mu m$-diameter channels occasionally using a 17.3-MHz focused transducer in water $\left(20^{\circ} \mathrm{C}, \lambda \approx 86 \mu \mathrm{m}\right.$, total sample thickness 220 $\mu m)$. However, interaction between ultrasound and sample microstructure - the underlying detection mechanism is poorly understood. Experimental evidence showed that the subwavelength channel was fused inside the two binding trilaminate plastic package films. Each trilaminate film had three sublayers. Package sample impedance profiles along the ultrasound beam axis were examined. Although identical in nominal impedance properties before sealing, the two binding films showed an asymmetric impedance profile after sealing. A generalized impedance profile model was proposed. The defect detection behavior of the echo signal was investigated by solving the 2D linear acoustic wave equations in fluid with finite-difference time-domain method and the perfectly matched layer absorbing boundary. The normalized correlation coefficients between the simulated and the measured RF echo waveforms were greater than $95 \%$ for this generalized model.
\end{abstract}

\section{INTRODUCTION}

The ultrasonic pulse-echo Backscattered Amplitude Integral (BAI)-mode imaging technique has been developed to detect small channel defects embedded in flexible food package seals [1]. The BAI-mode image is constructed with the BAI-values, values that are determined at each transducer sampling position by taking the area under the envelope of the received backscattered echo. This technique has demonstrated sensitive subwavelength defect detection capability, e.g., $100 \%$ detection of water-filled channels as small as 38 $\mu m$ in diameter with a $17.3-\mathrm{MHz}$ focused (f/2) transducer for the tested samples (cylindrical channels in bonded 2sheet plastic package samples, thickness $110 \mu \mathrm{m}$ per sheet) in water $\left(20^{\circ} \mathrm{C}, c \approx 1485 \mathrm{~m} / \mathrm{s}, \lambda \approx 86 \mu \mathrm{m}\right)$. Moreover,

Supported by the C-FAR program, University of Illinois

${ }^{1}$ Email correspondence to xyin@uiuc.edu. water-filled channel defects as small as $6 \mu \mathrm{m}$ in diameter could be intermittently detected.

The channel defect package sample was produced by using a heat sealer to fuse a smooth, die-drawn tungsten wire in between two plastic package films and removing the wire along its axial direction after the package sample had cooled. Each trilaminate package film has three sublayers (from inner sealing surface to outer surface): polypropylene $\left(80 \mu \mathrm{m}, c=2660 \mathrm{~m} / \mathrm{s}, \rho=900 \mathrm{~kg} / \mathrm{m}^{3}\right)$, polyvinylidene chloride $\left(15 \mu \mathrm{m}, c=2380 \mathrm{~m} / \mathrm{s}, \rho=1650 \mathrm{~kg} / \mathrm{m}^{3}\right)$, and oriented nylon $\left(15 \mu \mathrm{m}, c=2600 \mathrm{~m} / \mathrm{s}, \rho=1140 \mathrm{~kg} / \mathrm{m}^{3}\right)$. A water-filled channel defect was then created by immersing the fused plastic sample in degassed water. Thus, the channel defect package sample has one cylindrical channel defect embedded in six sublayers, having "sandwich-like" layered structure.

Although the method has been proven to be reliable for defect detection, the underlying detection mechanism is poorly understood. Ultrasound beam encounters complex structures in its propagation path: the package sample front wall, interfaces between sublayers, heat-fusing zone between the two plastic sheets, the channel itself, impedance deviation surrounding the channel, and the package sample back wall. The water-filled channel represents a small scatterer size and a minimal impedance difference. The backscattered echoes caused by the channel defect are weaker than the reflected echoes caused by the front and back walls of the plastic package. Furthermore, the echoes from the channel defect are sometimes buried in the echoes from the multiple reflections caused by the package sample. Thus, a theoretical modeling study has been undertaken to investigate the origin of the BAI-mode image contrast, and hopefully establish a generalized numerical model for the pulse-echo subwavelength defect detection system. The Finite-Difference Time-Domain (FDTD) method with Perfectly Matched Layer (PML) absorbing boundary was used to numerically evaluate the aforementioned interactions between ultrasound beam and sample microstructure. FDTD is good at simulating transient wave propagation and scattering phenomena. PML absorbs the outgoing wave at the truncated computational domain so that a simulated free space can be achieved [2]. 


\section{Methodology}

Simulation of the ultrasonic pulse-echo defect detection behavior has been implemented by solving the linear acoustic wave equations in a homogeneous, lossy fluid medium using FDTD with PML. The equations are

$$
\begin{aligned}
\nabla p(\mathbf{r}, t) & =-\rho \frac{\partial}{\partial t} \mathbf{u}(\mathbf{r}, t)-\alpha^{*} \mathbf{u}(\mathbf{r}, t) \\
\nabla \cdot \mathbf{u}(\mathbf{r}, t) & =-\kappa \frac{\partial}{\partial t} p(\mathbf{r}, t)-\alpha p(\mathbf{r}, t)
\end{aligned}
$$

where $p$ is the acoustic pressure field, $\mathbf{u}$ is the vector velocity field, $\mathbf{r}$ is the vector field point, $t$ is time, $\rho$ is the mass density of the medium, $\kappa$ is the compressibility of the medium, $\alpha$ is the attenuation coefficient associated with compressibility, and $\alpha^{*}$ is the attenuation coefficient associated with mass density. The absorption in the PML is achieved by setting $\alpha^{*}=\alpha \rho / \kappa$ in PML region and $\alpha^{*}=0$ in the acoustic medium [2].

The measured transducer center frequency is $17.3 \mathrm{MHz}$. The diameter and the characterized focal length of the spherically focused transducer (Panametrics V317) are $6.35 \mathrm{~mm}$ and $12.44 \mathrm{~mm}$ respectively, which are $74 \lambda$ and $145 \lambda$ in degassed water. It is computationally inefficient to include the transducer and the entire transducer-to-sample wave propagation path in the FDTD computational domain.

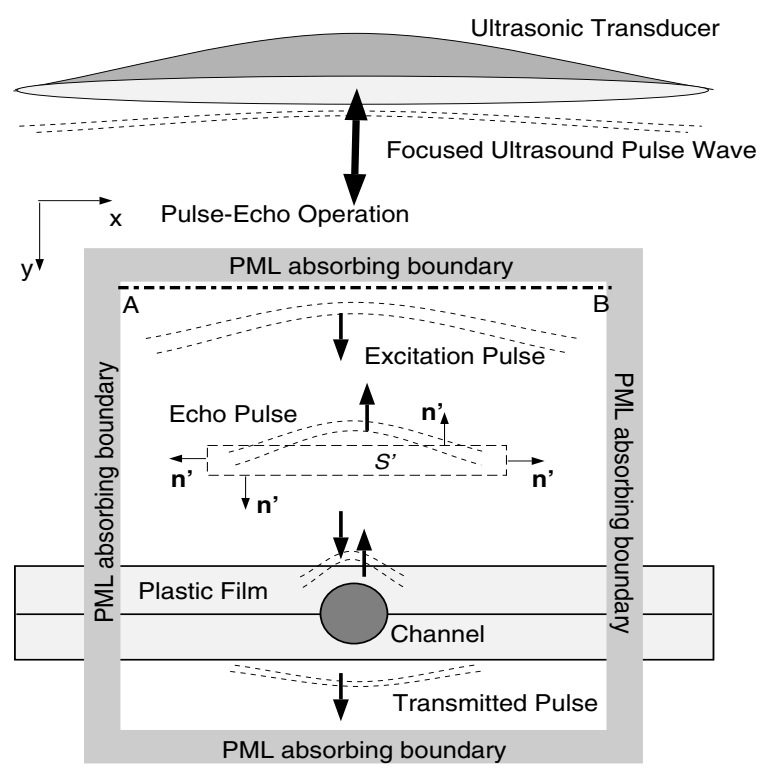

Figure 1: Pulse-echo simulation with FDTD and PML

To reduce domain size, the $2 \mathrm{D}$ simulation uses FIELDII [3] to calculate the transmitted ultrasound pulse pressure field along the line $\mathrm{AB}$ (Figure 1). The ultrasound field along line $\mathrm{AB}$ (Figure 2) is equivalent to the pulsed ultrasound wave that reaches this line in the FDTD simulation. The

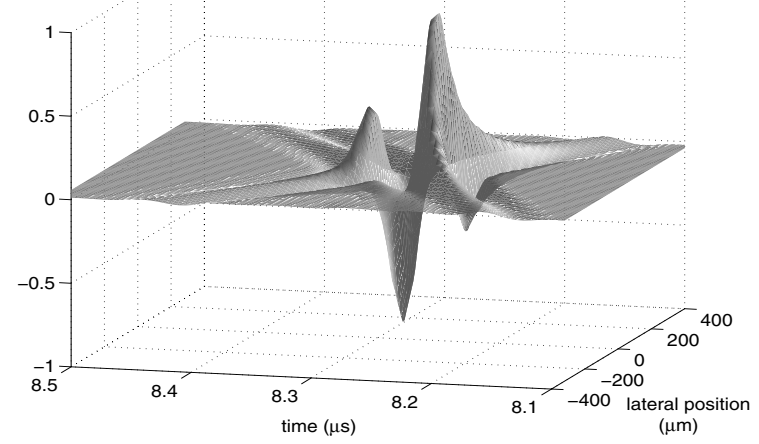

Figure 2: Emitted lateral pressure field along line AB

transmitted pulse is simulated by

$$
p_{\text {inc }}(t)=\frac{t}{T} \exp \left(-4 \Delta f^{2} t^{2}\right) \sin \left(2 \pi f_{0} t\right)
$$

where $f_{0}=17.3 \mathrm{MHz}$ (center frequency), $T=2.5 / f_{0}$ (pulse duration of the transmitted pulse), and $\Delta f=7.35 \mathrm{MHz}(-3-$ $\mathrm{dB}$ bandwidth).

The echo pressure received by the transducer surface is extrapolated by evaluating the transient Helmholtz integral (3) along the enclosed contour ( $S^{\prime}$ in Figure 1) [4].

$$
\begin{array}{r}
p(\mathbf{r}, t)=\frac{1}{2 \pi} \oint_{s^{\prime}}\left[\frac { \partial r } { \partial \mathbf { n } ^ { \prime } } \left(\frac{\tilde{p}\left(t-\frac{r}{c}\right)}{r^{2}}\right.\right. \\
\left.+\frac{1}{c r} \frac{\partial \tilde{p}\left(t-\frac{r}{c}\right)}{\partial t}\right) \\
\left.+\frac{1}{r} \frac{\partial \tilde{p}\left(t-\frac{r}{c}\right)}{\partial \mathbf{n}^{\prime}}\right] d S^{\prime}
\end{array}
$$

where $\mathbf{n}^{\prime}$ is the outward surface norm on $S^{\prime}$, and $c$ is the speed of sound in medium. The enclosed surface $S^{\prime}$ consists of evenly divided radiating elements whose positions are $\left(x^{\prime}\right.$, $\left.y^{\prime}\right)$. The transducer surface is partitioned with equally sized receiving elements whose positions are $(x, y)$. The distance between each radiating-receiving pair is $\mathbf{r}=\hat{i}\left(x-x^{\prime}\right)+$ $\hat{j}\left(y-y^{\prime}\right)$. The pressure $\tilde{p}\left(t-\frac{r}{c}\right)$ on $S^{\prime}$ is computed by the FDTD simulation at each time step.

The computational domain is significantly reduced from $145 \lambda$ long by $47 \lambda$ wide to $10 \lambda$ by $10 \lambda$ by using FIELD-II and the transient Helmholtz integral. The 2D simulation in Cartesian coordinates using a rectangular grid FDTD with PML has been implemented in MATLAB. The FDTD evolution uses a spatial-temporal update scheme that is similar to the well-known Yee algorithm [5]. To ensure stability, the grid size $\Delta=\lambda_{\min } / 20$ and the time evolution step $h=\Delta / 2 c_{\max }$, where $\lambda_{\min }$ is the minimal wavelength and $c_{\max }$ is the maximum speed of sound in the computational domain. The PML has a 10-grid thickness. 


\section{SAMPlE MicRostruCtURE}

An impedance profile model has to be established for the pulse-echo defect detection simulation. The impedance profile of interest is the cross-section profile of the package sample in the 2D simulation. A package sample was cut perpendicularly along its planar surface. Two steps were involved in measuring the impedance profile: (i). A focused transducer $(37.4 \mathrm{MHz},-6-\mathrm{dB}$ pulse-echo focal beam width of $52.9 \mu \mathrm{m}$ ) scanned a flat plexiglass surface whose impedance is known (reference echo). (ii). The transducer scanned the package sample cross section (220- $\mu \mathrm{m}$ thickness) at 5- $\mu m$ step size, with beam axis parallel to package sample planar surface. The echo amplitude at each scan location was compared with the reference echo to determine the impedance at each scan location.

An optical microscopic image of the package sample cross section was obtained (Figure 3). Several measured sample impedance profiles along the ultrasound wave propagation path were examined. Two impedance profiles at two randomly chosen horizontal cuts are shown (Figure 3). Although identical in nominal impedance properties before sealing, the two binding films showed an asymmetric impedance profile after sealing, with the maximum impedance occurring near the front wall of the package sample. The multiple reflections in the asymmetric sublayers behaved with more complexity than in the presumed symmetric sublayers.
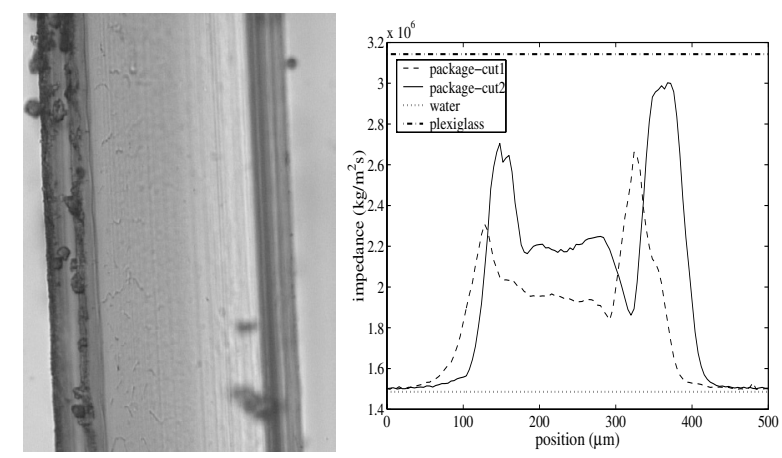

Figure 3: Optical microscopic image (left) and two impedance profile curves (right) of a package sample

The package sample with a water-filled channel defect was also examined (Figure 4). The channel defect was not exactly centered between the walls. Position shift was observed in the image. The shape of the channel defect was not a perfect circle as the shape of the tungsten wire. It appeared to be an ellipse shape and the contour was not smooth, demonstrating an impedance perturbation region surrounding the channel defect.

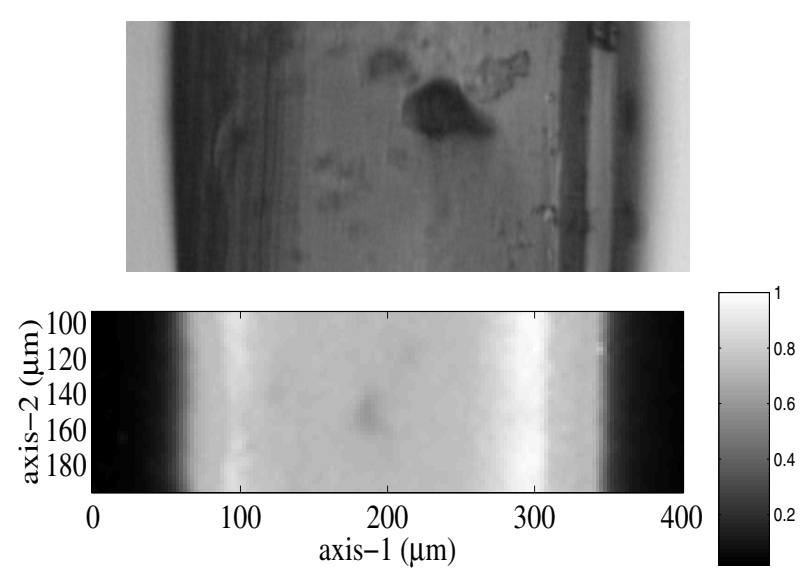

Figure 4: Optical microscopic image (top) and acoustic impedance image (bottom) of a package sample containing 38- $\mu m$-diameter water-filled channel

\section{Results}

Based on the nominal impedance properties and the microstructure measurement of the package sample, a generalized impedance profile was proposed. Figure 5 shows two impedance profiles along the pulse-echo beam axis. The impedance map was from a package sample with a 38- $\mu m$ diameter channel defect. The ultrasonic beam came from the right in both figures. The solid line corresponded to the intact region. The dashed line corresponded to a cut through the center of the defect. The channel defect was modeled as an ellipse with a 38- $\mu m$ major axis and a 30- $\mu m$ minor axis. The minor axis was parallel with the beam axis. The defect was filled with water and there was a $20-\mu m$-thick surrounding annulus region whose impedance was a bit higher than that of the intact region. The non-smooth channel contour was constructed by inwardly or outwardly shifting a small position offset of 0-6 $\mu \mathrm{m}$ randomly on the smooth elliptic contour. The channel defect had a $30-\mu m$ position shift toward the front wall of the package sample.
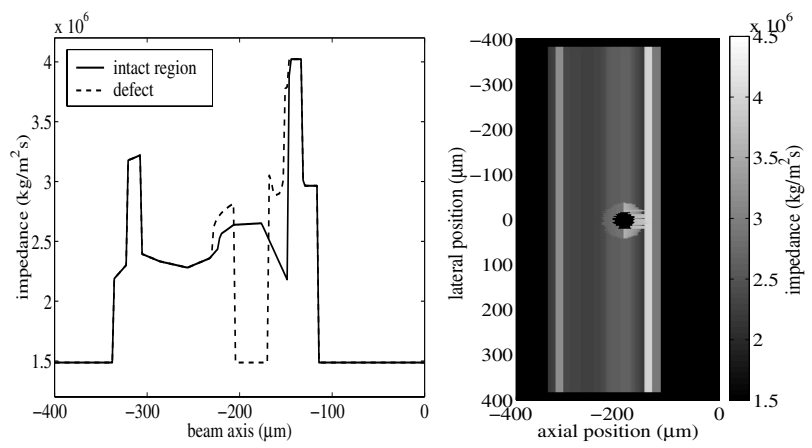

Figure 5: Generalized impedance model, left: impedance profile, right: defect sample impedance map 
With this generalized impedance profile model, simulated echoes were obtained (Figures 6 and 7). The normalized cross-correlation coefficient $R$ between the simulated and measured echo waveforms was calculated as a quantitative index of similarity. The simulated and measured results matched very well ( $R=97.5 \%$ for no defect situation and $R$ $=95.8 \%$ for defect situation).
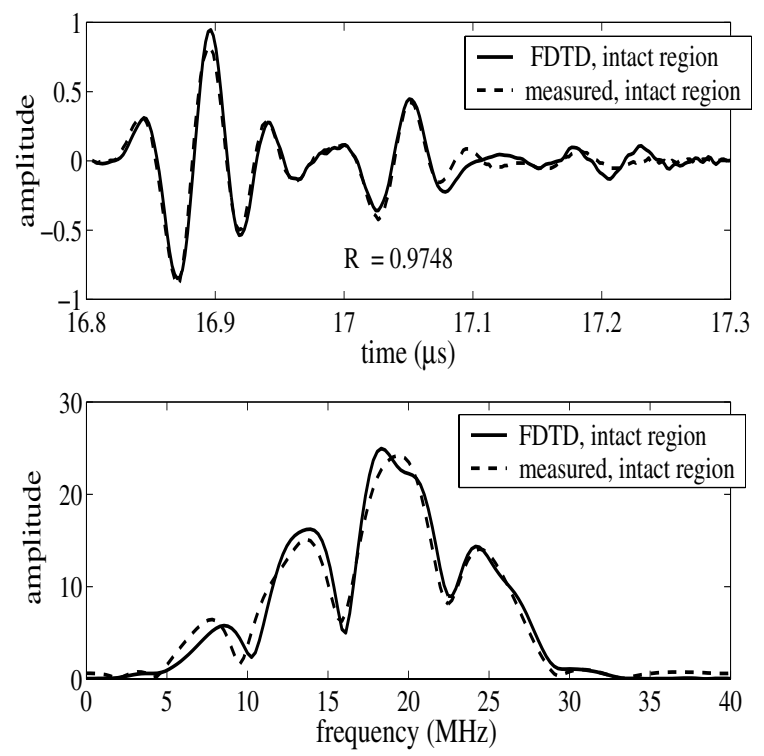

Figure 6: Echo waveform comparison between simulation and experiment, intact region, time domain (top) and frequency domain (bottom)

\section{CONCLUSION AND Future WORK}

The pulse-echo subwavelength channel defect detection has been simulated with FDTD and PML. A generalized impedance profile model was proposed. The simulation results showed good agreement with experiment results, which validated the impedance profile model. Future work will be conducted to study how the model parameters could affect the echo waveform, such as position of the defect, contour shape of the defect, bonding region between two package films, etc... A thorough study of the parameters will increase our knowledge on the nondestructive pulseecho subwavelength defect detection mechanism.

\section{ACKNOWLWDGEMENTS}

The authors would like to thank Dr. James Zachary and Mr. James Blue for their assistance in preparing the optical microscopic images of the plastic food package samples.
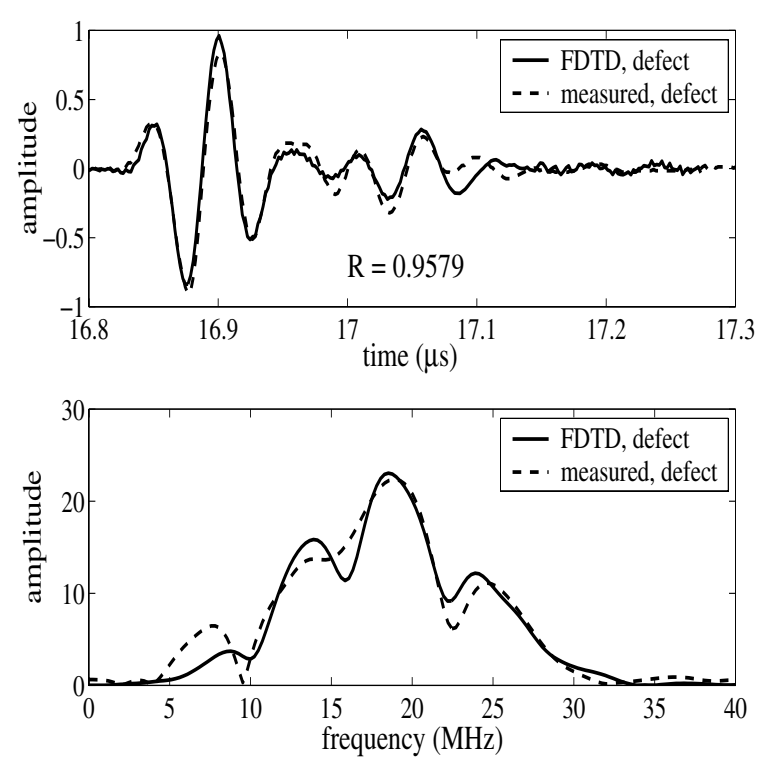

Figure 7: Echo waveform comparison between simulation and experiment, channel defect, time domain (top) and frequency domain (bottom)

\section{REFERENCES}

[1] K. Raum, A. Ozguler, S. A. Morris and W. D. O'Brien, Jr. "Channel defect detection in shelf-stable food packages using high-frequency pulse-echo imaging," IEEE Trans. Ultrason., Ferroelect., Freq. Contr., vol. 45, no. 1, pp. 30-40, 1998.

[2] X. Yuan, D. Borup, J. W. Wiskin, M. Berggren, R. Eidens and S. A. Johnson, "Formulation and validation of Berenger's PML absorbing boundary for the FDTD simulation of acoustic scattering," IEEE Trans. Ultrason., Ferroelect., Freq. Contr., vol. 44, No. 4, pp. 816-822, 1997.

[3] J. A. Jensen, "User's guide for the FIELD-II program," version 2.70 of May 26, 1999, with URL: $<$ http://www.es.oersted.dtu.dk/ jaj/field/>.

[4] R. Lerch, H. Landes and H. T. Kaarmann, "Finite element modeling of the pulse-echo behavior of ultrasound transducers," Proc. IEEE Int. Ultrason. Symp., pp. 1021-1025, 1994.

[5] K. S. Yee, "Numerical solution of initial boundary value problems involving Maxwell's equations in isotropic media," IEEE Trans. Antennas Propagat., vol. 14, No. 3, pp. 302-307, 1966. 